\title{
Time Lens based Optical Fourier Transformation for All-Optical Signal Processing of Spectrally-Efficient Data
}

Guan, Pengyu; Røge, Kasper Meldgaard; Lillieholm, Mads; Galili, Michael; Hu, Hao; Morioka, Toshio; Oxenløwe, Leif Katsuo

Published in:

Journal of Lightwave Technology

Link to article, DOI:

10.1109/JLT.2016.2614186

Publication date:

2017

Document Version

Peer reviewed version

Link back to DTU Orbit

Citation (APA):

Guan, P., Røge, K. M., Lillieholm, M., Galili, M., Hu, H., Morioka, T., \& Oxenløwe, L. K. (2017). Time Lens based Optical Fourier Transformation for All-Optical Signal Processing of Spectrally-Efficient Data. Journal of Lightwave Technology, 35(4), 799 - 806. [7579624]. https://doi.org/10.1109/JLT.2016.2614186

\section{General rights}

Copyright and moral rights for the publications made accessible in the public portal are retained by the authors and/or other copyright owners and it is a condition of accessing publications that users recognise and abide by the legal requirements associated with these rights.

- Users may download and print one copy of any publication from the public portal for the purpose of private study or research.

- You may not further distribute the material or use it for any profit-making activity or commercial gain

- You may freely distribute the URL identifying the publication in the public portal 


\title{
Time Lens based Optical Fourier Transformation for All-Optical Signal Processing of Spectrally-Efficient Data
}

\author{
P. Guan, Member, IEEE, K. M. Røge, M. Lillieholm, M. Galili, Member, OSA, H. Hu, \\ T. Morioka, Member, IEEE, Fellow, OSA, and L. K. Oxenløwe, Member, OSA
}

\begin{abstract}
We review recent progress in the use of time lens based optical Fourier transformation for advanced all-optical signal processing. A novel time lens based complete optical Fourier transformation (OFT) technique is introduced. This complete OFT is based on two quadratic phase-modulation stages using four-wave mixing (FWM), separated by a dispersive medium, which enables time-to-frequency and frequency-to-time conversions simultaneously, thus performing an exchange between the temporal and spectral profiles of the input signal. Using the proposed complete OFT, several advanced all-optical signal processing schemes for spectrally-efficient systems and networks have been achieved, including all-optical generation, detection and format conversion of spectrally-efficient signals. The spectrally-efficient signals in this paper mainly refer to efficiently multiplexed signals with a high symbol rate per $\mathrm{Hz}$, such as orthogonal frequency division multiplexing (OFDM), Nyquist wavelength-division multiplexing (Nyquist-WDM) and Nyquist optical time division multiplexing (Nyquist-OTDM) signals.
\end{abstract}

Index Terms - All-optical OFDM, optical Fourier transformation, OTDM, Nyquist-WDM, optical signal processing.

\section{INTRODUCTION}

$\mathrm{D}$ ue to the rapid traffic growth in optical communication networks, current infrastructures and technologies may not be able to handle the coming increase in data traffic and associated energy requirements [1-2]. Thus, it has become increasingly important to develop energy- and spectrally-efficient communication systems and the associated enabling technologies. One possible solution is the combination of several lower-rate channels with high spectral efficiency (SE) using orthogonal frequency division

Manuscript received $\mathrm{xx}-\mathrm{xx}-\mathrm{xxxx}$; revised $\mathrm{xx}$; accepted $\mathrm{xx}$. Date of publication $\mathrm{xx}$; date of current version $\mathrm{xx}$. This work was funded by the Danish Research Council FTP project LENS-COM (ref. DFF-5054-00184), FTP-TOR project (ref.no. 0602-02398B), the DFF Sap. Aude Adv. Grant NANO-SPECs (DFF-4005-00558B) and the DNRF Research Centre, SPOC (ref. DNRF123).

P. Guan, K. M. Røge, M. Galili, H. Hu, M. Lillieholm, T. Morioka and L. K. Oxenløwe are with DTU Fotonik, Department of Photonics Engineering, Technical University of Denmark, Ørsteds Plads 343, DK-2800 Kgs. Lyngby, Denmark (e-mail: pengu@fotonik.dtu.dk).

Color versions of one or more of the figures in this paper are available online at http://ieeexplore.ieee.org.

Copyright (c) 2016 IEEE
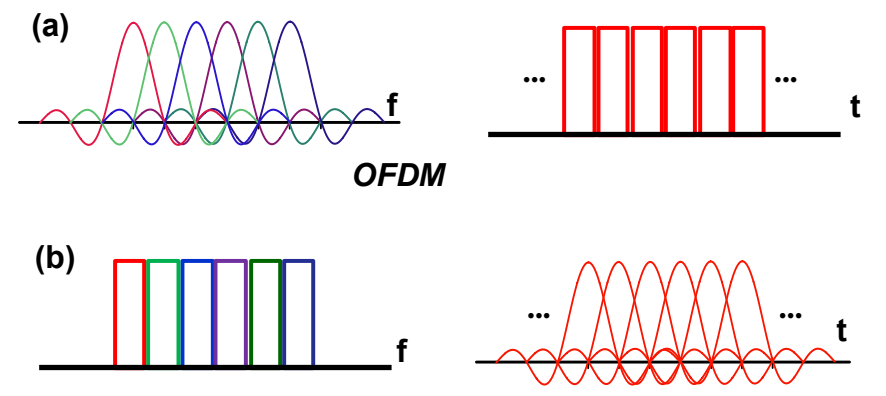

Nyquist WDM

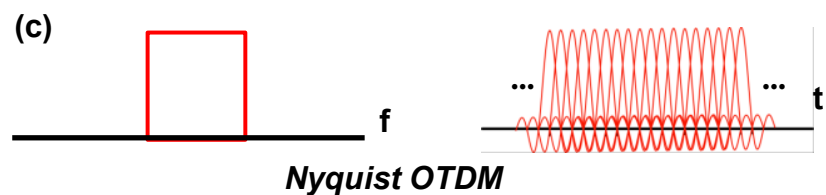

Fig. 1. Spectrum and corresponding waveform of: (a) OFDM, (b) Nyquist-WDM and (c) Nyquist-OTDM signals.

multiplexing (OFDM) [3], Nyquist wavelength division multiplexing (Nyquist-WDM) [4] and Nyquist optical time division multiplexing (Nyquist-OTDM) [5], which all take advantage of the orthogonality to enable a channel bandwidth close or equal to the symbol rate. Fig. 1(a) illustrates the spectrum of an OFDM signal and the waveform of a selected OFDM subcarrier. It consists of closely spaced and overlapping sinc-shaped subcarriers in the frequency domain. The waveforms for each subcarrier are square-shaped and closely spaced. Fig. 1(b) illustrates the spectrum of a Nyquist-WDM signal and the waveform of a selected Nyquist WDM channel. It consists of closely spaced but non-overlapping individual channels in the frequency domain. For each Nyquist-WDM channel, the waveforms are sinc-shaped and overlap in time domain. Fig. 1(c) illustrates the spectrum and waveform of a Nyquist-OTDM signal. It consists of a single square-shaped channel with broad bandwidth in the frequency domain. Its waveforms are sinc-shaped and overlap in time domain as the Nyquist-WDM signal, but usually with a higher symbol rate (achieved by OTDM). All the three multiplexing techniques have the same performance in terms of spectrum efficiency. In particular, OFDM has a large tolerance to fiber dispersion, Nyquist-WDM is more robust to practical implementation constraints [4], and Nyquist-OTDM can offer ultrahigh symbol rate beyond electronic limitation, but with less network flexibility due to its inseparable broad spectrum. With the help 
of advanced coherent detection and electronic digital signal processing (DSP) technologies, OFDM, Nyquist-WDM and Nyquist-OTDM have been used to demonstrate high SE super-channels with more than $1 \mathrm{Tbit} / \mathrm{s}$ capacity [6]-[8]. However, due to the bandwidth limitation of electronics, scaling the bandwidth of the DSP based system will also scale the number of components and their combined power consumption. On the other hand, optical signal processing (OSP) that can avoid optical to electrical to optical (OEO) conversion, has the potential to realize energy-efficient systems [9]. In addition, OSP explores nonlinear optical phenomena which have a response time on the order of femtoseconds, thus allowing for a processing bandwidth beyond one THz. In general, OSP is considered suitable only for relatively simple functionalities, and complex digital logic for spectrally-efficient signals may turn out to be too challenging to implement all-optically.

In this paper, we describe a novel time lens based complete optical Fourier transformation (OFT) technique for advanced optical signal processing of OFDM, Nyquist-OTDM and Nyquist-WDM signals. A number of recent demonstrations of complex high-speed optical signal processing using complete OFT will also be reviewed, including generation, detection and format conversion of OFDM, Nyquist-WDM and Nyquist-OTDM signals. These demonstrations show that the simple OFT implementations can be used to achieve relatively complex signal processing operations.

In section II, the principle of time lens based complete OFT is described and discussed. In section III, recent experimental demonstrations and results are presented. In section IV, we discuss the potential and the future implications and finally conclude this paper.

\section{PRINCIPLE Of TIME LENS BASED COMPLETE OFT}

The time lens is based on the space-time duality of light, which states that a quadratic phase modulation (PM) of a temporal waveform is analogous to the action of a thin lens on a spatial beam, hence the expression "time lens" [10]. By combining a time lens with suitable dispersion media both before and after the lens, an OFT can be realized as shown in Fig. 2(a) [11]. Recently, the time lens based OFT has been shown to be a very versatile and powerful tool for ultrafast optical signal processing. It can be applied to transfer the temporal profile of an optical signal into the frequency domain and vice-versa. This has led to demonstrations of e.g. distortion-less transmission [12], an ultrafast optical oscilloscope [13] and optical time division multiplexing (OTDM) to WDM conversion [14]. One common feature of these demonstrations is that they are all realized by a "partial" OFT, where only one-way conversion can be achieved, either from the time- to the frequency-domain, or from the frequencyto the time-domain. A partial OFT scheme is not sufficient for processing of OFDM, Nyquist-WDM and Nyquist-OTDM signals, especially at the transmitter, since it introduces a residual chirp or dispersion which will impact the orthogonality of OFDM and Nyquist-WDM signals. To overcome this challenge, we proposed a new time lens based
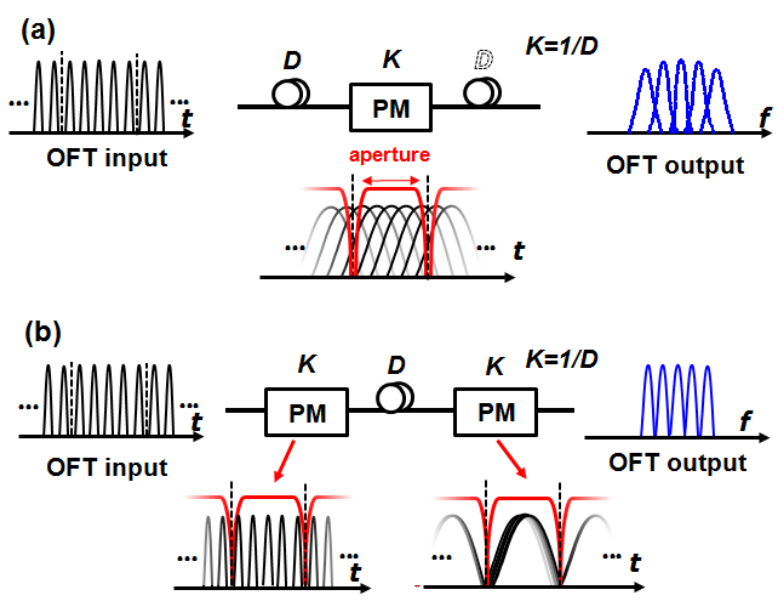

Fig. 2. Schematic diagrams of a time lens based OFT: (a) a traditional D-K-D configuration and (b) a proposed K-D-K configuration for signal processing of spectrally-efficient signals.

complete OFT, cf. Fig. 2(b) [15]. The proposed OFT is based on two quadratic phase-modulation stages $\left(\delta \phi=K t^{2} / 2\right)$ with chirp rate $K$, separated by a dispersive medium with $D=\beta_{2} L$ (where $\beta_{2}$ is the 2 nd order dispersion and $L$ is the length), which satisfies the condition $K=1 / D$ (a $K-D-K$ configuration). Consider an input signal waveform $u(t)$. The output waveform $v(t)$ after the first phase modulation stage and the dispersive medium is given by:

$$
v(t)=\sqrt{\frac{i}{2 \pi D}} \int_{-\infty}^{\infty} u\left(t^{\prime}\right) \exp \left(i \frac{K}{2} t^{\prime 2}\right) \exp \left[-\frac{i}{2 D}\left(t-t^{\prime}\right)^{2}\right] d t^{\prime} .
$$

When $K=1 / D$, the expression can be simplified to the following form that contains the Fourier transform of the input signal,

$$
v(t)=\sqrt{\frac{i}{2 \pi D}} \exp \left(-i \frac{K}{2} t^{2}\right) \int_{-\infty}^{\infty} u\left(t^{\prime}\right) \exp \left[i\left(\frac{t}{D}\right) t^{\prime}\right] d t^{\prime} .
$$

After chirp compensation in the second phase modulation stage, the temporal output becomes a scaled version of the spectral input:

$$
v(t)=\sqrt{\frac{i}{2 \pi D}} U\left(\frac{t}{D}\right)
$$

Taking the Fourier transform of (3) then yields the output spectrum $V(\omega)$ :

$$
V(\omega)=\sqrt{i 2 \pi D} u(-\omega D),
$$

which is a scaled version of the temporal input. Unlike the partial OFT schemes, this configuration enables TABLE I

COMPARISON OF DIFFERENT OFT CONFIGURATIONS

\begin{tabular}{c|ccc}
\hline \hline $\begin{array}{c}\text { OFT } \\
\text { Configurations }\end{array}$ & $\begin{array}{c}\text { Achievable } \\
\text { conversion(s) }\end{array}$ & $\begin{array}{c}\text { Residual } \\
\text { chirp or } \\
\text { dispersion }\end{array}$ & $\begin{array}{c}\text { Guard interval } \\
\text { requirement }\end{array}$ \\
\hline $\mathrm{D}-\mathrm{K}$ & Time $\rightarrow$ Frequency & Dispersion & Large \\
\hline $\mathrm{K}-\mathrm{D}$ & Frequency $\rightarrow$ Time & Chirp & small \\
\hline $\mathrm{D}-\mathrm{K}-\mathrm{D}$ & $\begin{array}{l}\text { Time } \rightarrow \text { Frequency } \\
\text { Frequency } \rightarrow \text { Time }\end{array}$ & No & Large \\
\hline $\mathrm{K}-\mathrm{D}-\mathrm{K}$ & $\begin{array}{l}\text { Frequency } \rightarrow \text { Time } \\
\text { Time } \rightarrow \text { Frequency }\end{array}$ & No & small \\
\hline
\end{tabular}


time-to-frequency and frequency-to-time conversions simultaneously, thus performing an exchange between the temporal and spectral profiles of the input signal (a "complete" OFT) without any residual chirp or dispersion impacting the output signal. Note that the traditional OFT system with a $D-K-D$ configuration in Fig. 2(a) can also realize simultaneous frequency-to-time and time-to-frequency conversion. However, it is not suitable for processing signals with high SE, since the dispersive elements before the phase modulation stage will generally broaden the input waveform beyond its time slot, causing temporal clipping and hence spectral broadening and power loss of the edge channels after OFT. Thus, large inter-channel crosstalk and OSNR degradation occurs. To avoid these degradations, a large temporal guard interval (GI) is essential, at the cost of reduced SE. In contrast, the proposed OFT with a $K-D-K$ configuration pre-chirps the input signals at the first phase modulation stage. When the chirped signals propagate in the dispersive medium, they will not only experience waveform broadening, but also become aligned (focused) to the center of the second phase-modulation stage. This configuration thus confines the waveform to the time-lens apertures, allowing for a complete OFT with a small GI. Table I compares different OFT configurations, indicating that the $K-D-K$ configuration is more versatile for signal processing of spectrally-efficient signals.

\section{RECENT DEMONSTRATIONS OF ADVANCED ALL-OPTICAL SIGNAL PROCESSING USING TIME LENS BASED OFT}

In this section, a number of representative demonstrations of complex optical signal processing using complete OFT will be reviewed, with a focus on all-optical generation, detection and format conversion of spectrally-efficient signals.

\section{A. All-optical OFDM transmitters}

All-optical OFDM performs subcarrier multiplexing and demultiplexing in the optical domain, thereby circumventing the speed limitations of electronics to enable a significantly larger capacity [16]. The all-optical OFDM approach is also attractive since digital/analog conversion and DSP are avoided both for the multiplexing at the transmitter and the

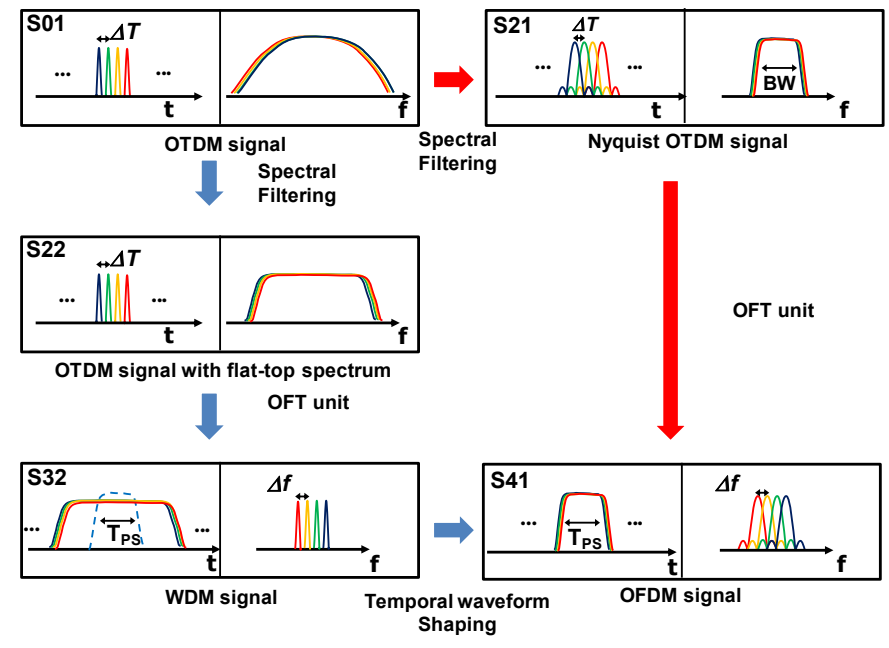

Fig. 3. Principle of OFDM signal generation based on time-domain OFT from an OTDM signal, using two equivalent approaches (red and blue).

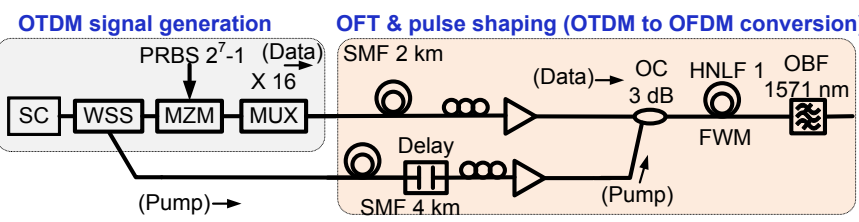

Fig. 4. Experimental setup for 160 Gbaud all-optical OFDM signal generation using time lens based OFT.

demultiplexing at the receiver, allowing for simpler, lower cost and less energy-consuming transmitter/receiver pairs. Most all-optical OFDM transmitters use rectangular-like electrical modulation to generate the OFDM subcarriers from individual comb-lines, and then combine them using a coupler [6], [16]. However, such transmitters are limited by the electronics bandwidth in realizing the fast symbol transitions of OFDM subcarriers. To avoid this limitation, the OFDM subcarriers can also be generated from data-modulated optical pulses using optical discrete Fourier transformation (ODFT) based on cascaded delay interferometers [17], arrayed waveguide grating routers [18], or wavelength selective switches (WSS) [19]. However, the complexity of these ODFT schemes generally increases with the number of independent data-subcarriers that must be generated. As an alternative, a novel all-optical OFDM signal generation scheme utilizing time lens based OFT has been proposed [20], [21]. With this scheme, all of the OFDM subcarriers are simultaneously generated in real-time from an OTDM signal. The principle is shown in Fig. 3. The OFDM signal can be generated from an OTDM signal through two different approaches. In one approach (indicated by red arrows), the OTDM signal is converted with tributary spacing $\Delta T$ to a Nyquist-OTDM signal, by first using a rectangular spectral filter, and then exchanging the temporal and spectral envelopes of the Nyquist-OTDM signal using the proposed complete OFT. This results in an OFDM signal with subcarrier spacing $\Delta f=K \cdot \Delta T / 2 \pi$. The other approach follows the blue arrows, where the spectrum of the OTDM signal is filtered into a flat-top shape using a WSS, and then converted to a WDM signal (S32) using the complete OFT. If the input pulse width $\delta t_{0}$ is narrow enough such that the temporal Fraunhofer condition $\left|\delta t_{0}{ }^{2} / 2 D\right|<<1$ is satisfied, then the first phase modulation stage can be omitted [22], leading to the simpler $D-K$ configuration. A rectangular pulse shaper with gate-width $T_{P S}$ is then used to select the temporal center of the WDM signal, resulting in an OFDM signal with subcarrier spectral width given by $1 / T_{P S}$, set equal to $\Delta f$ to achieve frequency orthogonality. Note that the complete OFT is only achieved within the finite time aperture $T_{P S}$ in the D-K configuration. Furthermore, a guard interval is introduced between each group of OTDM tributaries so that the pulse tails from neighboring bit slots within the gate-window are mapped to wavelengths well separated from the desired OFDM spectrum. These wavelengths are finally removed by an optical bandpass filter (OBF). Compared to other ODFT schemes, the system complexity does not increase with the number of subcarriers.

In the experimental demonstration, a 16-subcarrier, $160 \mathrm{Gbit} / \mathrm{s}$ OFDM signal is generated all-optically through the 
(a)

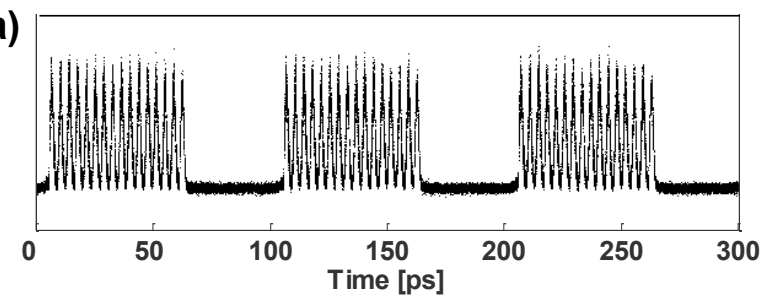

(b)

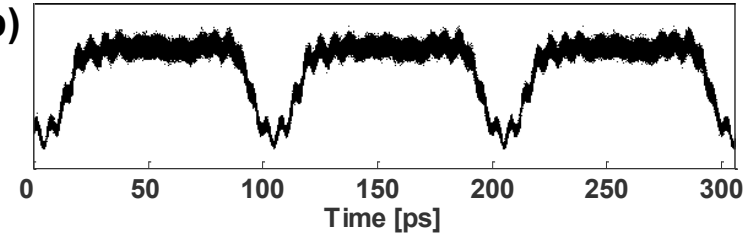

Fig. 5. (a) The waveform of the $160 \mathrm{Gbit} / \mathrm{s}$ OTDM DPSK signal, (b) and the waveform of the chirped rectangular pump pulses for FWM.

blue path. The experimental setup is show in Fig. 4. An SC signal is generated by a $10 \mathrm{GHz}$ mode-locked laser and a $400 \mathrm{~m}$ dispersion-flattened highly non-linear fiber (DF-HNLF). The SC signal is filtered into $1.7 \mathrm{ps}$ wide pulses with a flat-top spectrum using a WSS, which then undergoes DPSK modulation and OTDM multiplexing, resulting in a $160 \mathrm{Gbit} / \mathrm{s}$ OTDM-DPSK signal centered at $1551 \mathrm{~nm}$ with a guard interval of $44 \mathrm{ps}$ between each group of the 16 tributaries. The guard interval is generated optically as part of the OTDM multiplexing. The waveform of the $160 \mathrm{Gbit} / \mathrm{s}$ OTDM DPSK signal is shown in Fig. 5(a). The generated OTDM data signal is launched into a complete OFT for OTDM to OFDM conversion. A simple D-K configuration is used for complete OFT as the
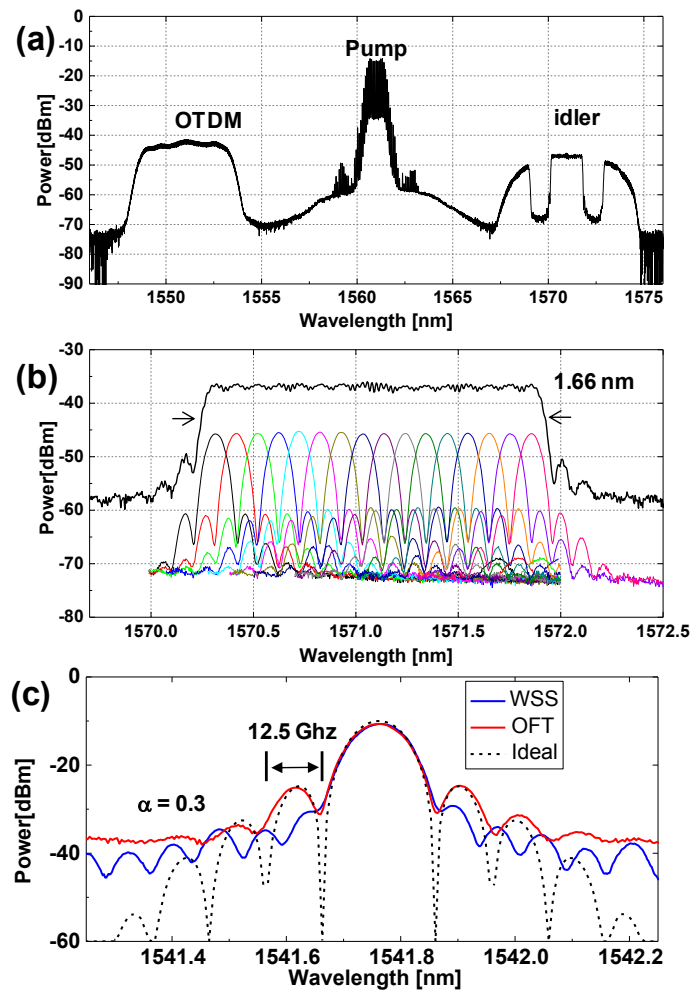

Fig. 6. Results of all-optical OFDM generation: (a) output spectrum of the FWM, (b) obtained OFDM spectrum and individual sinc-shaped subcarriers for different input OTDM tributaries, (c) spectrum of a single $12.5 \mathrm{GHz}$ sinc subcarrier, a subcarrier generated by WSS and an ideal sinc shape with $\alpha=0.3$. input pulse width is narrow enough to satisfy the condition $\left|\delta t_{0}^{2} / 2 D\right|<<1$. The quadratic phase modulation is implemented based on a four-wave mixing (FWM) process in a highly nonlinear fiber (HNLF) using linearly chirped rectangular pump pulses [13]. As the first step of the OFT process, the 160 Gbit/s OTDM signal is dispersed in $2 \mathrm{~km} \mathrm{SSMF,} \mathrm{resulting} \mathrm{in}$ overlapping $160 \mathrm{ps}$ flat-top linearly-chirped pulses. The quadratic phase modulation and pulse shaping are simultaneously achieved in a FWM process (HNLF1) using $80-p s$ linearly chirped rectangular pump pulses $(1561 \mathrm{~nm})$, obtained through filtering by a WSS and propagation in $4 \mathrm{~km}$ SSMF. Fig. 5(b) shows the waveform of the chirped rectangular pump pulses. Fig. 6(a) shows the spectrum after FWM. The center part of the idler is the 16-subcarrier OFDM spectrum with $12.5 \mathrm{GHz}$ channel spacing. The empty bands in the idler spectrum originate from the 44 ps guard-intervals in the OTDM signal. A zoom-in of the obtained OFDM spectrum is shown in Fig. 6(b). Spectra obtained for individual OFDM subcarrier are also shown in Fig. 6(b), where the characteristic sinc-shape can be observed. The spectrum of a single $12.5 \mathrm{GHz}$ sinc subcarrier is shown in Fig. 6(c). To compare with one of the most proven all-optical approaches [19], a subcarrier generated by a WSS (Finisar Waveshaper) that has been used for $28 \times 10 \mathrm{Gbit} / \mathrm{s}$ all optical OFDM generation [24] is shown, in addition to an ideal sinc profile with roll-off $\alpha=0.3$. It can be seen that the spectrum out of the WSS is distorted due to the limited frequency resolution, whereas a close to ideal $12.5 \mathrm{GHz}$ OFDM subcarrier is obtained using OFT, indicating a better performance for the generation of narrow OFDM subcarriers.

In addition, it has been proposed that an OFDM signal can also be generated all-optically from a WDM signal [21]. The principle is shown in Fig. 7. As described above, an OFDM signal can be generated from either a Nyquist-OTDM signal (S21) or an OTDM signal with a flat-top spectrum (S22). These two kinds of signals (S21, S22) can in turn be generated from a WDM signal (S02) using an extra OFT, and thus two additional approaches to generate an OFDM signal are obtained. One (indicated by red arrows) is intermediately converting a WDM signal to a Nyquist-OTDM signal (S21) using a rectangular temporal waveform shaper followed by a complete OFT, and then converted to an OFDM signal using another OFT. This

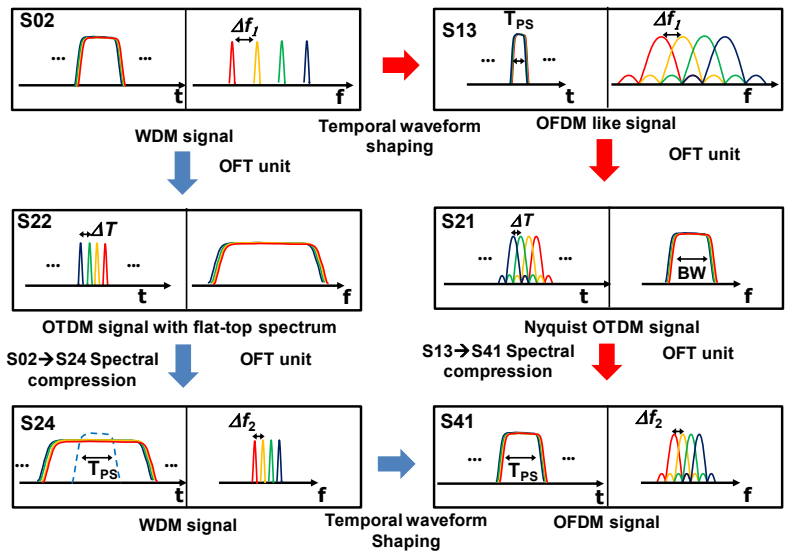

Fig. 7. Principle of OFDM signal generation based on time-domain OFT from a WDM signal using two equivalent approaches (red and blue). 


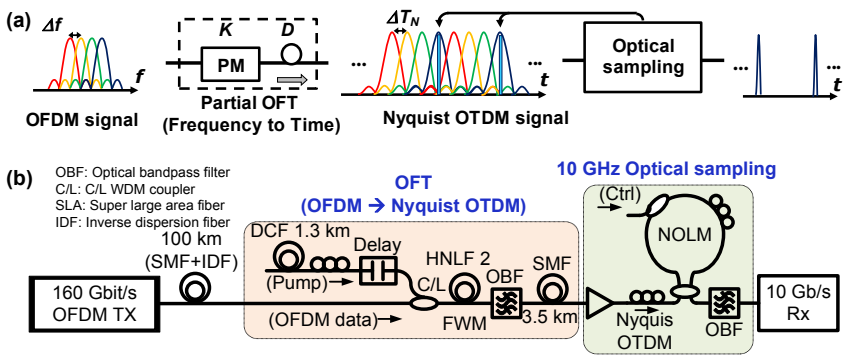

Fig. 8. (a) Principle of all-optical OFDM demultiplexing using time-lens based OFT, (b) experimental setup of a 160 Gbaud all-optical OFDM receiver using time lens based OFT.

approach can also be described as rectangular temporal waveform shaper followed by a spectral compressor. The other one (indicated by blue arrows) is intermediately converting a WDM signal to an OTDM signal with a flat-top spectrum (S22) using a complete OFT, and then converted to an OFDM signal using another OFT, followed by a rectangular temporal waveform shaper. This approach can also be described as a spectral compressor followed by a rectangular temporal waveform shaper. These schemes may provide simple approaches that can greatly enhance the spectral efficiency of existing commercial WDM systems.

\section{B. All-optical OFDM receivers}

The subcarriers of the OFDM signal are equidistantly spaced, thus overlapping with a frequency null-point at all other subcarriers as shown in Fig. 1(a). For the demultiplexing, it is important to note that the individual OFDM subcarrier cannot simply be extracted by optical bandpass filtering as in traditional WDM systems, since this would imply a large penalty due to the crosstalk from the spectrally overlapping neighbor subcarriers. Previously the OFDM subcarriers were demultiplexed either in the electrical domain using DSP [3], or in the optical domain using ODFT based on various structures of optical splitters, delays, phase-shifters and time-gates
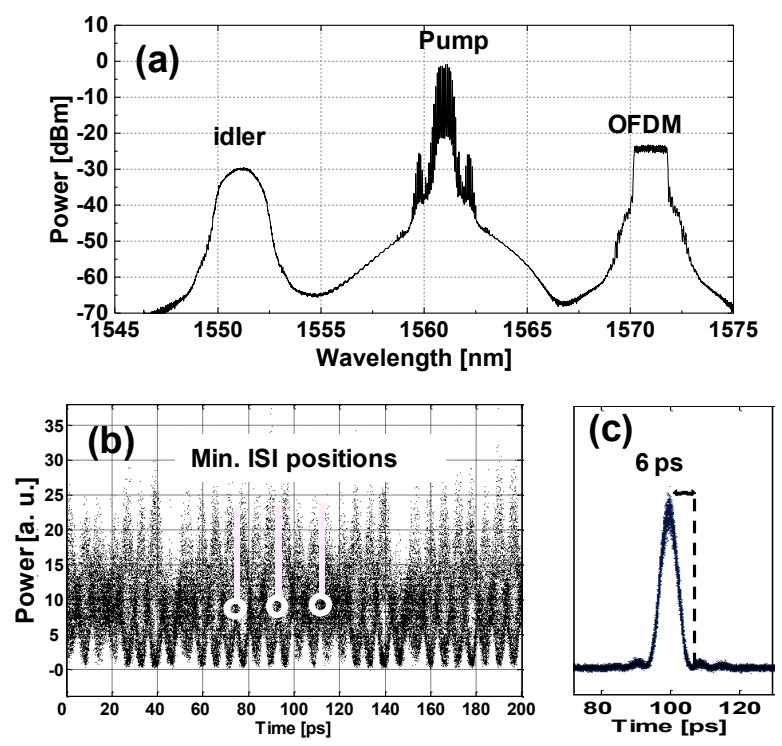

Fig. 9. Results of all-optical OFDM demultiplexing, (a) output FWM spectrum of HNLF 2, (b) obtained Nyquist-OTDM like signal, (c) and the waveform of a single Nyquist tributary.
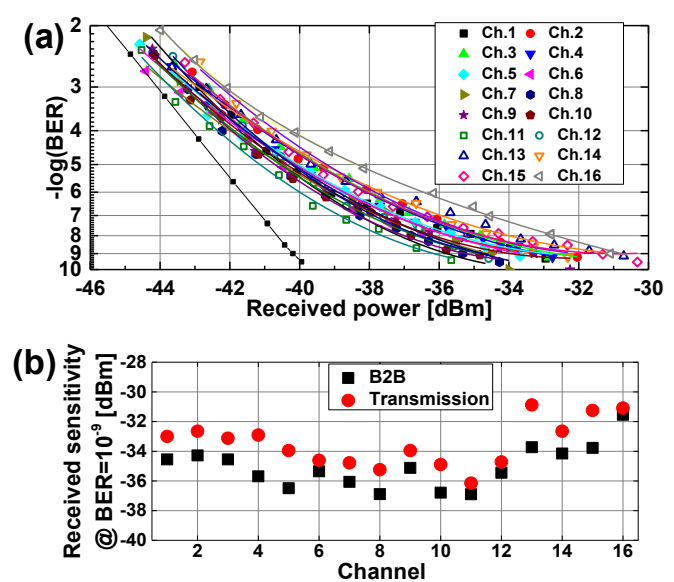

Fig. 10. (a) BER measurements of 16 OFDM subcarriers (10 Gbit/s DPSK) after $100 \mathrm{~km}$ transmission, (b) Receiver power sensitivities at $\mathrm{BER}=10^{-9}$ B2B and after $100 \mathrm{~km}$.

[17]-[19]. In this section, we introduce a new all-optical OFDM demultiplexing scheme using time lens based OFT [20]. The principle is shown in Fig. 8(a). To receive an OFDM signal, a partial time lens based OFT is sufficient to frequency-to-time convert the OFDM signal into a Nyquist-OTDM like signal, which has sinc-shaped waveforms and non-square spectrum. The Nyquist-OTDM like signal is subsequently time-demultiplexed by sampling each subcarrier at the intersymbol interference (ISI) free point using a narrow optical sampling gate [5]. Finally, the demultiplexed signal is detected using a base rate receiver.

In the experimental demonstration, a 16-subcarrier $160 \mathrm{Gbit} / \mathrm{s}$ OFDM signal is obtained from an OFT based all optical OFDM transmitter that has been introduced in section 3A. The experimental setup is shown in Fig. 8(b). The generated $160 \mathrm{Gbit} / \mathrm{s}$ OFDM signal was transmitted over a $100 \mathrm{~km}$ dispersion-managed fiber-link consisting of super large area fiber (SLA) and inverse dispersion fiber (IDF). In the receiver, a time lens based OFT, achieved in a FWM process (HNLF 2), is used for the OFDM to Nyquist-OTDM conversion, and a nonlinear optical loop mirror (NOLM) is employed for the $10 \mathrm{GHz}$ optical sampling using $1.1 \mathrm{ps}$ wide control pulses. The FWM pump pulses $(1561 \mathrm{~nm})$ and NOLM control-pulses $(1566 \mathrm{~nm})$ are obtained via spectral broadening in DF-HNLFs and spectral filtering. The output FWM spectrum of HNLF 2 is shown in Fig. 9(a). The idler at $1551 \mathrm{~nm}$ is extracted by an OBF and propagated through $3.6 \mathrm{~km}$ SSMF, resulting in the 160 Gbaud Nyquist-OTDM like signal as shown in Fig. 9(b). The tributary spacing is $6 \mathrm{ps,} \mathrm{and} \mathrm{the}$ sinc-shaped waveform of a single tributary can be observed in Fig. 9(c). The ISI-free position of each tributary is sampled out using the NOLM, and the BER of each tributary (subcarrier) is measured in a 10-Gbit/s pre-amplified DPSK receiver. The BER performance is shown in Fig. 10(a). The 100-km $160 \mathrm{Gbit} / \mathrm{s}$ OFDM transmission is successful with BER $<10^{-9}$ performance for all 16 subcarriers. Fig. 10(b) shows the receiver sensitivities of all channels at $\mathrm{BER}=10^{-9}$ for the 100-km transmission, and for the back-to-back (B2B) system. The average transmission penalty is $1.6 \mathrm{~dB}$, which is mainly attributed to additional jitter in the clock recovery from the OFDM signal. 


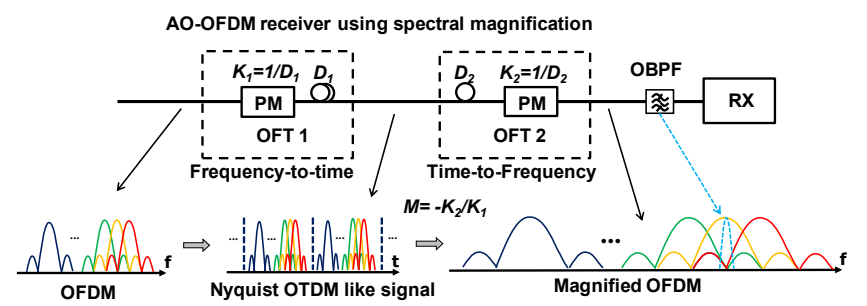

Fig. 11. Principle of an all-optical OFDM receiver using time lens based spectral magnification and optical bandpass filtering.

Furthermore, another all-optical OFDM demultiplexing scheme using time lens based spectral magnification has been demonstrated [23], which allows for the direct detection of all subcarriers without using temporal sampling gates, further reducing the complexity of the system. The principle is shown in Fig. 11. The first OFT converts the OFDM spectrum to a Nyquist-OTDM like signal (frequency-to-time conversion), and the second OFT converts it back to the spectral domain (time-to-frequency conversion). The magnification factor is determined by the ratio of the two employed chirp rates $K$. The magnified spectrum enables reduced intercarrier interference when using OBFs to extract the subcarriers. This scheme has been used for all-optical demultiplexing of a 28 -subcarrier, 280 Gbit/s OFDM signal [24].

\section{Conversion from DWDM to Nyquist-OTDM}

The Nyquist-OTDM technique takes advantage of time domain orthogonality, allowing the signal bandwidth to be reduced to the symbol rate without ISI, enabling terabit-per-second transmission with high SE [8]. Recently, a novel dense WDM (DWDM) to a single Nyquist channel (Nyquist-OTDM) conversion scheme based on complete OFT and optical Nyquist filtering has been proposed [25]. The principle is shown in Fig. 12(a). The DWDM channels with flat-top temporal waveforms are bit-wise synchronized. A complete OFT using a K-D-K configuration is then applied to exchange the temporal and spectral profiles of the input DWDM signal, resulting in an OTDM signal with tributary spacing of $\Delta T=2 \pi \Delta f / K$. To achieve maximum SE, the OTDM

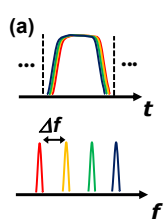

DWDM signal
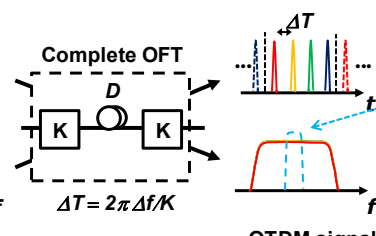

OTDM signal

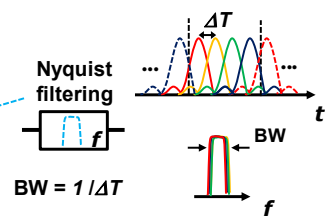

Nyquist OTDM channe
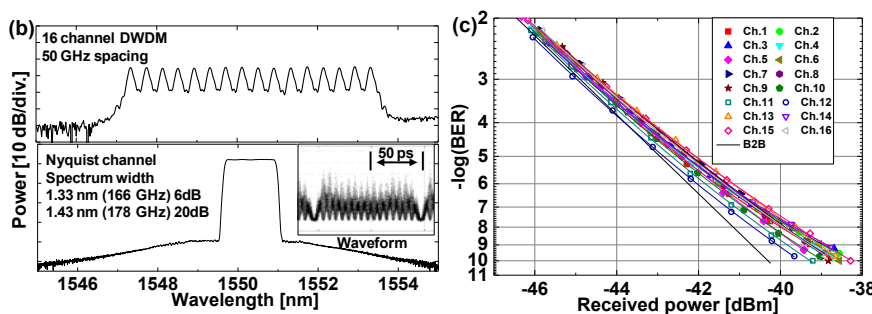

Fig. 12. (a) Principle of DWDM signal to a single Nyquist channel conversion, (b) conversion from a 16x10 Gbit/s DPSK, 50-GHz spacing DWDM signal to a $160 \mathrm{Gbit} / \mathrm{s}$ Nyquist channel with $166 \mathrm{GHz}$ total bandwidth, inset is the temporal waveform, (c) BER measurements for all 16 demultiplexed Nyquist-OTDM tributaries. channel spacing $\Delta T$ is set equal to $1 / f_{\mathrm{s}}$, where $f_{\mathrm{s}}$ is the OTDM symbol rate. A rectangular OBF (Nyquist filtering) with bandwidth $B W=1 / \Delta T$ is then used to filter the center of the overlapping spectra, resulting in a Nyquist-OTDM signal with sinc-shaped tributaries. In the experimental demonstration, a $16 \times 10 \mathrm{Gbit} / \mathrm{s}$ DPSK, $50-\mathrm{GHz}$ spacing DWDM signal was successfully converted to a $160 \mathrm{Gbit} / \mathrm{s}$ Nyquist-OTDM signal with $166 \mathrm{GHz}$ total bandwidth as shown in Fig. 12(b), thus increasing the SE from 0.2 to $0.96 \mathrm{symbol} / \mathrm{s} / \mathrm{Hz}$. This indicates
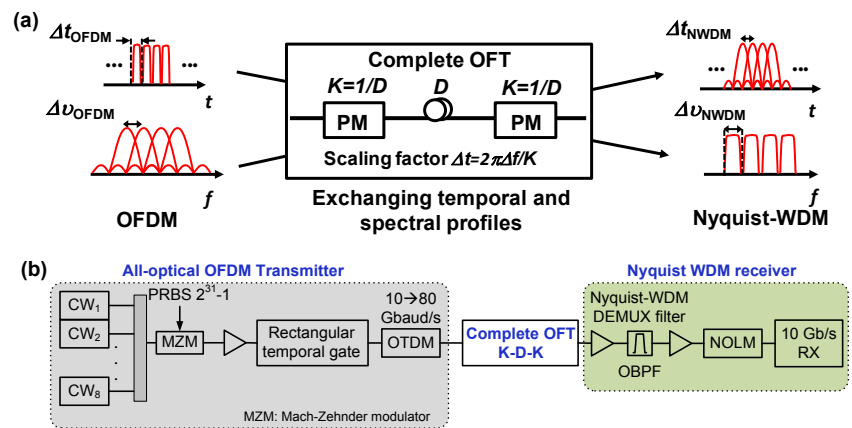

Fig.13. (a) The principle of all-optical OFDM to Nyquist-WDM conversion, (b) and experimental setup for conversion of an 8-subcarrier $640 \mathrm{Gbit} / \mathrm{s}$ OFDM super-channel to eight $80-$ Gbit/s Nyquist-WDM channels.

that the total transmission capacity can be improved by a factor of $\sim 5$. The Nyquist-OTDM signal is subsequently received by optical sampling using a NOLM based narrow time-gate. Fig. 12(c) shows the BER measurements for all 16 demultiplexed Nyquist-OTDM tributaries, where error-free operation $\left(\mathrm{BER}<10^{-9}\right)$ is achieved for all tributaries. The average power penalty was only $1.4 \mathrm{~dB}$ for the full system. This technique has the potential to provide a simple and economical solution to upgrade commercial DWDM systems.

\section{Conversion from OFDM to Nyquist-WDM}

Spectrally-efficient signals such as OFDM, Nyquist-WDM and Nyquist-OTDM have been intensely investigated for next generation commercial communication systems. If employed side by side, it may be very important to have an efficient tool for converting signals between these systems. However, such functionality has not previously been possible unless complex OEO conversion and DSP are used, which may further increase the complexity, power-consumption and cost. Recently, a novel all-optical ultra-high-speed OFDM to Nyquist-WDM conversion scheme based on complete OFT has been proposed [15]. This scheme is essentially simple, and the implementation complexity does not scale with the number of OFDM subcarriers. The principle is shown in Fig. 13(a). As the OFDM and Nyquist-WDM are equivalent when exchanging the temporal and spectral profiles, they are the ideal candidates for complete OFT processing. The experimental setup is shown in Fig. 13(b). An 8-subcarrier $640 \mathrm{Gbit} / \mathrm{s}$ differential phase-shift keying (DPSK) OFDM super-channel is generated by an all-optical OFDM transmitter, based on rectangular temporal gating and OTDM technique. A complete OFT with a K-D-K configuration is used for the OFDM to Nyquist-WDM conversion. In the receiver, the eight converted $80-\mathrm{Gbit} / \mathrm{s}$ 


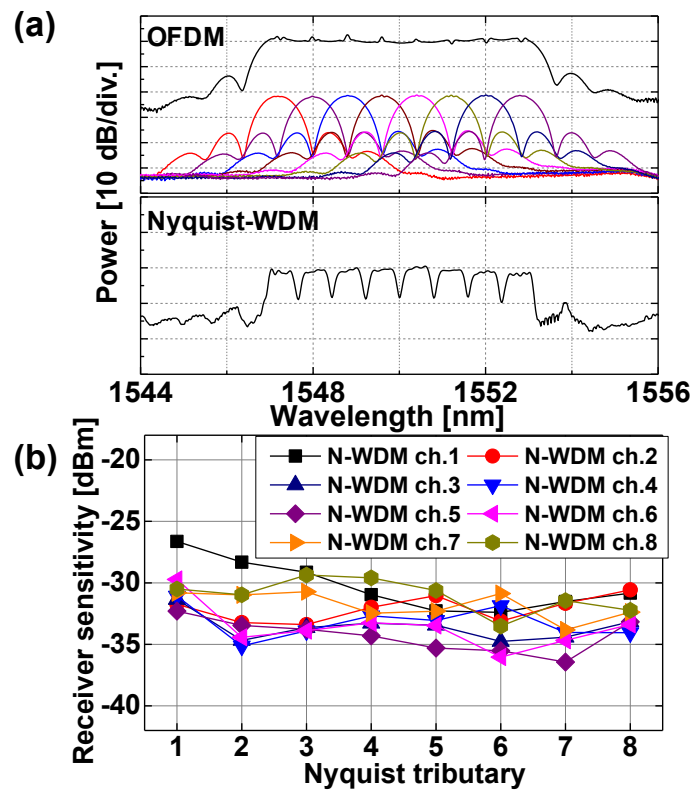

Fig.14. (a) Optical spectra of the original OFDM signal and individual subcarriers (top) and of the output Nyquist WDM signal (bottom), (b) receiver sensitivities at $\mathrm{BER}=10^{-9}$ of all converted Nyquist-WDM channels. Nyquist-WDM channels are received by WDM demultiplexing using a rectangular filter, followed by optical sampling using a NOLM. The experimental results are shown in Fig. 14. A $640 \mathrm{Gbit} / \mathrm{s}$ OFDM signal was successfully converted to eight Nyquist-WDM channels as shown in Fig. 14(a), achieving BER $<10^{-9}$ performance for all channels. Fig. 14(b) shows the corresponding receiver sensitivities at $\mathrm{BER}=10^{-9}$. The $\mathrm{SE}$ remains unchanged at $0.8 \mathrm{symbol} / \mathrm{s} / \mathrm{Hz}$ after conversion. To the best of our knowledge, this is the first and only demonstration of OFDM to Nyquist-WDM conversion. In principle, such an OFT-based technique can also be employed for Nyquist-WDM to OFDM conversion. It could therefore provide a simple and energy-efficient link between spectrally-efficient OFDM and Nyquist-WDM systems.

\section{DISCUSSIONS AND CONCLUSION}

Time lens based optical Fourier transformation has a wide range of applications, including distortion-less transmission, ultrafast optical oscilloscopes and optical format conversion between the time and frequency domains. In this paper, we have reviewed our recent progress in advanced all-optical processing of spectrally-efficient signals using time lens based OFT. A novel time lens based complete OFT technique for advanced processing of OFDM, Nyquist-WDM and Nyquist-OTDM signals has been introduced, and various demonstrations have been reviewed. Time lens based OFT has the benefit of enabling broadband ultrafast advanced OSP, which allows for the handling of a much larger number of channels, and also higher channel symbol rates in real-time. The implementation complexity does not increase with the number of channels, as opposed to the DSP approach. The same set-up can be used for any number of channels, as long as the total signal bandwidth can be accommodated by the time lenses.
This technique might enable even more complex signal processing applications, and become a very important enabling technology for future energy- and spectrally- efficient optical communication systems.

To realize higher spectrally-efficient communication system and networks, the capability to adapt the higher-level modulation format is very important. As the time lens based OFT in principle only exchanges the signal temporal and frequency profiles, the phase and amplitude information is preserved. Hence, the time lens is transparent to the data format. For example, a FWM-based OFT has been used to demonstrate OTDM-to-WDM conversion of a 320-GBd 16QAM signal [26]. Previously, time lenses have been demonstrated based on electro-optic phase modulation or nonlinear effects such as cross-phase modulation (XPM) or FWM [27]. The electro-optic approach provides a simpler configuration with lower loss, but the low PM index and the impurely quadratic phase modulation limits the performance of such a scheme. Using XPM based on parabolic pump pulses is the most straightforward all-optical approach to quadratic phase modulation, but the parabolic pump shape may become distorted due to self-phase modulation. The most suitable approach for spectrally efficient applications is FWM based on chirped pump pulses, which can achieve a large and purely quadratic phase-modulation. However, the phase noise of the pump is transferred to the signal in the FWM process, potentially limiting the performance for advanced signal modulation formats. Additionally, as a platform for time lens based OSP, nonlinear silicon photonics becomes more appealing, due to its integration potential with electronics, low cost, broad operation bandwidth and high space- and energyefficiency. Silicon photonic integration will make time lens based all-optical signal processing more attractive for the future energy- and spectrally-efficient communication systems.

\section{ACKNOWLEDGMENT}

The authors would like to thank OFS Denmark for supplying the nonlinear fibers and H. C. Hansen Mulvad for his great contributions. This work is supported by FTP project LENS-COM (ref. DFF-5054-00184), FTP-TOR project (ref.no. 0602-02398B), the DFF Sap. Aude Adv. Grant NANO-SPECs (DFF-4005-00558B) and the DNRF Research Centre, SPOC (ref. DNRF123).

\section{REFERENCES}

[1] Peter J. Winzer, "Scaling optical fiber networks: challenges and solutions," Optics \& Photonics News, vol. 26, no. 3, pp. 28-35, March 2015.

[2] T. Morioka, "New generation optical infrastructure technologies: "EXAT initiative" towards 2020 and beyond," in Proc. Opto Electronics and Communications Conference (OECC2009), paper FT4, July 2009.

[3] A. Lowery and J. Armstrong, "Orthogonal-frequency-division multiplexing for dispersion compensation of long-haul optical systems," Opt. Express, vol. 14, no. 6, pp. 2079-2084, March 2006.

[4] G. Bosco, V. Curri, A. Carena, P. Poggiolini, and F. Forghieri, "On the performance of Nyquist-WDM terabit superchannels based on 
PM-BPSK, PM-QPSK, PM-8QAM or PM-16QAM subcarriers," $J$. Lightwave Technol., vol. 29, no. 1, pp. 53-61, January 2011.

[5] M. Nakazawa, T. Hirooka, P. Ruan, and P. Guan, "Ultrahigh-speed "orthogonal" TDM transmission with an optical Nyquist pulse train," Opt. Express, vol. 20, no. 2. pp. 1129-1140, January 2012.

[6] X. Liu, S. Chandrasekhar, P. J. Winzer, T. Lotz, J. Carlson, J. Yang, G. Cheren, and S. Zederbaum, "1.5-Tb/s guard-banded superchannel transmission over 56x 100-km (5600-km) ULAF using 30-Gbaud pilot-free OFDM-16QAM signals with $5.75-\mathrm{b} / \mathrm{s} / \mathrm{Hz}$ net spectral efficiency," in Proc. European Conf. Optical Communication (ECOC2012), PDP, Th.3.C.5. September 2012.

[7] R. Cigliutti, A. Nespola, D. Zeolla, G. Bosco, A. Carena, V. Curri, F. Forghieri, Y. Yamamoto, T. Sasaki, and P. Poggiolini, "Ultra-long-haul transmission of $16 \times 112 \mathrm{~Gb} / \mathrm{s}$ spectrally-engineered DAC-generated Nyquist-WDM PM-16QAM channels with $1.05 x$ (symbol-rate) frequency spacing," in Proc. Optical Fiber Communication Conference, (OFC 2012), paper OTh3A3. March 2012.

[8] D. O. Otuya, K. Kasai, T. Hirooka, and M. Nakazawa, "Single-channel $1.92 \mathrm{Tbit} / \mathrm{s}, 64 \mathrm{QAM}$ coherent Nyquist orthogonal TDM transmission with a spectral efficiency of $10.6 \mathrm{bit} / \mathrm{s} / \mathrm{Hz}$," J. Lightwave Technol., vol. 34, no. 2, pp. 768-775, January 2016.

[9] H. C. H. Mulvad, E. Palushani, H. Hu, H. Ji, M. Galili, A. T. Clausen, P. Jeppesen, and L. K. Oxenløwe, "Recent advances in ultra-high-speed optical signal processing," in Proc. European Conf. Optical Communication (ECOC2012), paper Tu1 A5. September 2012.

[10] B. H. Kolner and M. Nazarathy, "Temporal imaging with a time lens," Opt. Lett., vol. 14, no. 12, pp. 630-632, June 1989.

[11] A. W. Lohmann and D. Mendlovic, "Temporal filtering with time lenses," Appl. Opt., vol. 31, no. 29, pp. 6212-6219, October 1992.

[12] M. Nakazawa, T. Hirooka, F. Futami and S. Watanabe, "Ideal distortion-free transmission using optical Fourier transformation and Fourier transform-limited optical pulses", IEEE Photon. Technol. Lett., vol. 16, no. 4, pp. 1059-1061, April 2004.

[13] M. A. Foster, R. Salem, D. F. Geraghty, A. C. Turner-Foster, M. Lipson, and A. L. Gaeta, "Silicon-chip-based ultrafast optical oscilloscope," Nature, vol. 456, pp. 81-84 September 2008.

[14] H. C. H. Mulvad, E. Palushani, M. Galili, J. Xu, H. Hu, A. Clausen, L. Oxenløwe, and P. Jeppesen, "OTDM-WDM conversion based on timedomain optical Fourier transformation with spectral compression", Optical Fiber Communication Conference, (OFC 2011), paper OThN2, March 2011.

[15] P. Guan, K. M. Røge, H. C. H. Mulvad, M. Galili, H. Hu, M. Lillieholm, T. Morioka, and L. K. Oxenløwe, "All-optical ultra-high-speed OFDM to Nyquist-WDM conversion based on complete optical Fourier transformation," J. Lightwave. Technol, vol. 34, no. 2, pp. 626-632, January 2016.

[16] D. Hillerkuss, R. Schmogrow, T. Schellinger, M. Jordan, M. Winter, G. Huber, T. Vallaitis, R. Bonk, P. Kleinow, F. Frey, M. Roeger, S. Koenig, A. Ludwig, A. Marculescu, J. Li, M. Hoh, M. Dreschmann, J. Meyer, S. Ben Ezra, N. Narkiss, B. Nebendahl, F. Parmigiani, P. Petropoulos, B. Resan, A. Oehler, K. Weingarten, T. Ellermeyer, J. Lutz, M. Moeller, M. Huebner, J. Becker, C. Koos, W. Freude, and J. Leuthold, "26 Tbit/s line-rate super-channel transmission utilizing all-optical fast Fourier transform processing," Nat. Photon., vol. 5, pp. 364-371, May 2011.

[17] D. Hillerkuss, M. Winter, M. Teschke, A. Marculescu, J. Li, G. Sigurdsson, K. Worms, S. Ben Ezra, N. Narkiss, W. Freude, and J. Leuthold, "Simple all-optical FFT scheme enabling Tbit/s real-time signal processing," Opt. Express, vol. 18, no. 9, pp. 9324-9340 April 2010.

[18] A. J. Lowery, and L. Du, "All-optical OFDM transmitter design using AWGRs and low-bandwidth modulators," Opt. Express vol. 19, no. 17, pp. 15696-15704, August 2011.

[19] J. Schröder, L. B. Du, J. Carpenter, B. J. Eggleton, and A. J. Lowery, "All-optical OFDM with cyclic prefix insertion using flexible wavelength selective switch optical processing," J. Lightwave Technol., vol. 32, no. 4, pp. 752-759, Feb. 2014.

[20] P. Guan, D. Kong, K. M. Røge, H. C. Hansen Mulvad, M. Galili, and L. K. Oxenløwe, "Real-time all-optical OFDM transmission system based on time-domain optical Fourier transformation." Optical Fiber Communication Conference (OFC 2014), paper W4F.1, March 2014.

[21] P. Guan, H.C. H. Mulvad, L. K. Oxenlowe, "All-optical orthogonal frequency division multiplexing (OFDM) transmitter" IPC No. H04J 14/ 00 A I. Patent No. EP2916472.

[22] E. Palushani, L. K. Oxenlowe, M. Galili, H. Mulvad, A. T. Clausen, and P. Jeppesen, "Flat-top pulse generation by the optical Fourier transform technique for ultrahigh speed signal processing," IEEE J. Quantum Electron. vol. 45, no. 11, pp. 1317-1324, November 2009.

[23] E. Palushani, H. C. Hansen Mulvad, D. Kong, P. Guan, M. Galili, and L.K. Oxenløwe, "All-optical OFDM demultiplexing by spectral magnification and band-pass filtering," Opt. Express vol. 22, no. 1, pp. 136-144 December 2014.

[24] P. Guan, S. Lefrancois, M. Lillieholm , H. C. Hansen Mulvad, K. M. Røge, H. Hu, J. Schroeder, B. J. Eggleton, Z. Geng, A. J. Lowery, T. Morioka, L. K. Oxenløwe, "All-optical OFDM system using a wavelength selective switch based transmitter and a spectral magnification based receiver." European Conference and Exhibition on Optical Communication (ECOC 2014), paper Tu.3.6.2, September 2014.

[25] P. Guan, K. M. Røge, H. C. Hansen Mulvad, H. Hu, T. Morioka, L. K. Oxenløwe, "Conversion of a DWDM signal to a single Nyquist channel based on a complete optical Fourier transformation." European Conference and Exhibition on Optical Communication (ECOC 2014), paper We.2.5.5, September 2014.

[26] E. Palushani, T. Richter, R. Ludwig, C. Schubert, H. C. H. Mulvad, A. Clausen, and L. Oxenløwe, "OTDM-to-WDM conversion of complex modulation formats by time-domain optical Fourier transformation," Optical Fiber Communication Conference, (OFC 2012), paper OTh3H.2. March 2012.

[27] R. Salem, M. A. Foster, and A. L. Gaeta, "Application of space-time duality to ultrahigh-speed optical signal processing," Adv. Opt. Photon., Vol. 5, no. 3, pp. 274-317, August 2013.

\section{Pengyu Guan}

\section{Kasper Meldgaard Røge}

\section{Mads Lillieholm}

\section{Michael Galili}

\section{Hao Hu}

\section{Toshio Morioka}

\section{Leif Katsuo Oxenløwe}

